\title{
IMT-1012 Immunotherapeutic Vaccine
}

National Cancer Institute

\section{Source}

National Cancer Institute. IMT-1012 Immunotherapeutic Vaccine. NCI Thesaurus. Code C77863.

A multi-peptide cancer vaccine with potential immunostimulating and antineoplastic activities. IMT-1012 immunotherapeutic vaccine contains twelve different synthetic peptides or tumor associated antigens (TAAs), including cyclin I (CCNI), cyclin-dependent kinase CDC2, EDDRI and TACE/ADAM17, each of which is involved in a different pathway associated with tumor growth, survival, and metastasis. Each antigen in the vaccine elicits a specific cytotoxic T-lymphocyte ( $C T L$ ) immune response against tumor cells expressing that antigen. This multi-antigen/multi-pathway targeting strategy provides broad immunotherapeutic coverage with respect to tumor complexity and heterogeneity and may result in enhanced vaccine efficacy. 